\title{
Search for multimessenger signals in NOvA coincident with LIGO/Virgo detections
}

M. A. Acero, ${ }^{2}$ P. Adamson, ${ }^{12}$ L. Aliaga, ${ }^{12}$ T. Alion, ${ }^{39}$ V. Allakhverdian, ${ }^{26}$ N. Anfimov ${ }^{26}$ A. Antoshkin, ${ }^{26}$ L. Asquith ${ }^{39}$ A. Aurisano, ${ }^{6}$ A. Back ${ }^{24}$ C. Backhouse, ${ }^{43}$ M. Baird,${ }^{20,39,44}$ N. Balashov, ${ }^{26}$ P. Baldi, ${ }^{25}$ B. A. Bambah, ${ }^{17}$ S. Bashar, ${ }^{42}$ K. Bays, ${ }^{4,19}$ S. Bending, ${ }^{43}$ R. Bernstein, ${ }^{12}$ V. Bhatnagar, ${ }^{32}$ B. Bhuyan, ${ }^{14}$ J. Bian, ${ }^{25,30}$ J. Blair, ${ }^{16}$ A. C. Booth, ${ }^{39}$ P. Bour, ${ }^{9}$ C. Bromberg, ${ }^{28}$ N. Buchanan, ${ }^{8}$ A. Butkevich, ${ }^{22}$ S. Calvez, ${ }^{8}$ T. J. Carroll, ${ }^{41,47}$ E. Catano-Mur, ${ }^{24,46}$ S. Childress, ${ }^{12}$ B. C. Choudhary, ${ }^{11}$ T. E. Coan,${ }^{37}$ M. Colo, ${ }^{46}$ L. Corwin, ${ }^{36}$ L. Cremonesi, ${ }^{43}$ G. S. Davies, ${ }^{31,20}$ P. F. Derwent, ${ }^{12}$ R. Dharmapalan, ${ }^{1}$ P. Ding, ${ }^{12}$ Z. Djurcic, ${ }^{1}$ D. Doyle, ${ }^{8}$ E. C. Dukes, ${ }^{44}$ P. Dung ${ }^{41}$ H. Duyang, ${ }^{35}$ S. Edayath ${ }^{7}$ R. Ehrlich, ${ }^{44}$ G. J. Feldman, ${ }^{15}$ P. Filip, ${ }^{23}$ W. Flanagan, ${ }^{10}$ M. J. Frank,${ }^{34}$ H. R. Gallagher, ${ }^{42}$ R. Gandrajula, ${ }^{28}$ F. Gao,${ }^{33}$ S. Germani, ${ }^{43}$ A. Giri, ${ }^{18}$ R. A. Gomes, ${ }^{13}$ M. C. Goodman, ${ }^{1}$ V. Grichine, ${ }^{27}$ M. Groh, ${ }^{20}$ R. Group, ${ }^{44}$ B. Guo, ${ }^{35}$ A. Habig, ${ }_{9}^{29}$ F. Hakl, ${ }^{21}$ J. Hartnell, ${ }^{39}$ R. Hatcher, ${ }^{12}$ K. Heller, ${ }^{30}$ J. Hewes, ${ }^{6}$ A. Himmel, ${ }^{12}$ A. Holin, ${ }^{43}$ J. Huang, ${ }^{41}$ J. Hylen, ${ }^{12}$ F. Jediny, ${ }^{9}$ C. Johnson, ${ }^{8}$ M. Judah ${ }^{8}$ I. Kakorin, ${ }^{26}$ D. Kalra, ${ }^{32}$ D. M. Kaplan, ${ }^{19}$ R. Keloth, ${ }^{7}$ O. Klimov, ${ }^{26}$ L. W. Koerner, ${ }^{16}$ L. Kolupaeva, ${ }^{26}$ S. Kotelnikov, ${ }^{27}$ Ch. Kullenberg, ${ }^{26}$ A. Kumar, ${ }^{32}$ C. D. Kuruppu, ${ }^{35}$ V. Kus, ${ }^{9}$ T. Lackey, ${ }^{20}$ K. Lang, ${ }^{41}$ L. Li, ${ }^{25}$ S. Lin, ${ }^{8}$ M. Lokajicek, ${ }^{23}$ S. Luchuk, ${ }^{22}$ S. Magill, ${ }^{1}$ W. A. Mann, ${ }^{42}$ M. L. Marshak, ${ }^{30}$ M. Martinez-Casales, ${ }^{24}$ V. Matveev, ${ }^{22}$ B. Mayes, ${ }^{39}$ D. P. Méndez, ${ }^{39}$ M. D. Messier, ${ }^{20}$ H. Meyer, ${ }^{45}$ T. Miao, ${ }^{12}$ W. H. Miller, ${ }^{30}$ S. R. Mishra, ${ }^{35}$ A. Mislivec, ${ }^{30}$ R. Mohanta, ${ }_{17}$ A. Moren, ${ }^{29}$ L. Mualem, ${ }^{4}$ M. Muether, ${ }^{45}$ S. Mufson, ${ }^{20}$ K. Mulder, ${ }^{43}$ R. Murphy, ${ }^{20}$ J. Musser, ${ }^{20}$ D. Naples ${ }^{33}$ N. Nayak ${ }^{25}$ J. K. Nelson, ${ }^{46}$ R. Nichol, ${ }^{43}$ E. Niner, ${ }^{12}$ A. Norman, ${ }^{12}$ A. Norrick, ${ }^{12}$ T. Nosek, ${ }^{5}$ A. Olshevskiy, ${ }^{26}$ T. Olson, ${ }^{42}$ J. Paley, ${ }^{12}$ R. B. Patterson, ${ }^{4}$ G. Pawloski, ${ }^{30}$ O. Petrova, ${ }^{26}$ R. Petti, ${ }^{35}$ R. K. Plunkett, ${ }^{12}$ A. Rafique, ${ }^{1}$ F. Psihas, ${ }^{20,41}$ V. Raj,${ }^{4}$ B. Rebel, ${ }^{12,47}$ P. Rojas, ${ }^{8}$ V. Ryabov, ${ }^{27}$ O. Samoylov, ${ }^{26}$ M. C. Sanchez,${ }^{24}$ S. Sánchez Falero, ${ }^{24}$ P. Shanahan, ${ }^{12}$ A. Sheshukov, ${ }^{26}$ P. Singh, ${ }^{11}$ V. Singh ${ }^{3}$ E. Smith, ${ }^{20}$ J. Smolik,${ }^{9}$ P. Snopok, ${ }^{19}$ N. Solomey, ${ }^{45}$ A. Sousa, ${ }^{6}$ K. Soustruznik, ${ }^{5}$ M. Strait, ${ }^{30,}{ }^{,}$ L. Suter, ${ }^{12}$ A. Sutton, ${ }^{44}$ R. L. Talaga, ${ }^{1}$ B. Tapia Oregui, ${ }^{41}$ P. Tas, ${ }^{5}$ R. B. Thayyullathil, ${ }^{7}$ J. Thomas ${ }^{43,47}$ E. Tiras,${ }^{24}$ D. Torbunov, ${ }^{30}$ J. Tripathi, ${ }^{32}$ Y. Torun, ${ }^{19}$ J. Urheim, ${ }^{20}$ P. Vahle,${ }^{46}$ J. Vasel, ${ }^{20}$ P. Vokac, ${ }^{9}$ T. Vrba, ${ }^{9}$ M. Wallbank, ${ }^{6}$ T. K. Warburton, ${ }^{24}$ M. Wetstein, ${ }^{24}$ D. Whittington, ${ }^{40,20}$ S. G. Wojcicki, ${ }^{38}$ J. Wolcott, ${ }^{42}$ A. Yallappa Dombara, ${ }^{40}$ K. Yonehara, ${ }^{12}$ S. Yu, ${ }^{1,19}$ Y. Yu, ${ }^{19}$ S. Zadorozhnyy, ${ }^{22}$ J. Zalesak, ${ }^{23}$ Y. Zhang, ${ }^{39}$ and R. Zwaska ${ }^{12}$

(NOvA Collaboration)

\author{
${ }^{1}$ Argonne National Laboratory, Argonne, Illinois 60439, USA \\ ${ }^{2}$ Universidad del Atlantico, Carrera 30 Number 8-49, Puerto Colombia, Atlantico, Colombia \\ ${ }^{3}$ Department of Physics, Institute of Science, Banaras Hindu University, Varanasi, 221 005, India \\ ${ }^{4}$ California Institute of Technology, Pasadena, California 91125, USA \\ ${ }^{5}$ Charles University, Faculty of Mathematics and Physics, Institute of Particle and Nuclear Physics, \\ Prague, Czech Republic \\ ${ }^{6}$ Department of Physics, University of Cincinnati, Cincinnati, Ohio 45221, USA \\ ${ }^{7}$ Department of Physics, Cochin University of Science and Technology, Kochi 682 022, India \\ ${ }^{8}$ Department of Physics, Colorado State University, Fort Collins, Colorado 80523-1875, USA \\ ${ }^{9}$ Czech Technical University in Prague, Brehova 7, 11519 Prague 1, Czech Republic \\ ${ }^{10}$ University of Dallas, 1845 E Northgate Drive, Irving, Texas 75062 USA \\ ${ }^{11}$ Department of Physics and Astrophysics, University of Delhi, Delhi 110007, India \\ ${ }^{12}$ Fermi National Accelerator Laboratory, Batavia, Illinois 60510, USA \\ ${ }^{13}$ Instituto de Física, Universidade Federal de Goiás, Goiânia, Goiás, 74690-900, Brazil \\ ${ }^{14}$ Department of Physics, IIT Guwahati, Guwahati, 781 039, India \\ ${ }^{15}$ Department of Physics, Harvard University, Cambridge, Massachusetts 02138, USA \\ ${ }^{16}$ Department of Physics, University of Houston, Houston, Texas 77204, USA \\ ${ }^{17}$ School of Physics, University of Hyderabad, Hyderabad, 500 046, India \\ ${ }^{18}$ Department of Physics, IIT Hyderabad, Hyderabad, 502 205, India \\ ${ }^{19}$ Department of Physics, Illinois Institute of Technology, Chicago, Illinois 60616, USA \\ ${ }^{20}$ Indiana University, Bloomington, Indiana 47405, USA \\ ${ }^{21}$ Institute of Computer Science, The Czech Academy of Sciences, 18207 Prague, Czech Republic \\ ${ }^{22}$ Inst. for Nuclear Research of Russia, Academy of Sciences 7a, 60th October Anniversary prospect, \\ Moscow 117312, Russia \\ ${ }^{23}$ Institute of Physics, The Czech Academy of Sciences, 18221 Prague, Czech Republic \\ ${ }^{24}$ Department of Physics and Astronomy, Iowa State University, Ames, Iowa 50011, USA \\ ${ }^{25}$ Department of Physics and Astronomy, University of California at Irvine, Irvine, California 92697, USA
}




\author{
${ }^{26}$ Joint Institute for Nuclear Research, Dubna, Moscow region 141980, Russia \\ ${ }^{27}$ Nuclear Physics and Astrophysics Division, Lebedev Physical Institute, \\ Leninsky Prospect 53, 119991 Moscow, Russia \\ ${ }^{28}$ Department of Physics and Astronomy, Michigan State University, East Lansing, Michigan 48824, USA \\ ${ }^{29}$ Department of Physics and Astronomy, University of Minnesota Duluth, Duluth, Minnesota 55812, USA \\ ${ }^{30}$ School of Physics and Astronomy, University of Minnesota Twin Cities, \\ Minneapolis, Minnesota 55455, USA \\ ${ }^{31}$ University of Mississippi, University, Mississippi 38677, USA \\ ${ }^{32}$ Department of Physics, Panjab University, Chandigarh, 160 014, India \\ ${ }^{33}$ Department of Physics, University of Pittsburgh, Pittsburgh, Pennsylvania 15260, USA \\ ${ }^{34}$ Department of Physics, University of South Alabama, Mobile, Alabama 36688, USA \\ ${ }^{35}$ Department of Physics and Astronomy, University of South Carolina, \\ Columbia, South Carolina 29208, USA \\ ${ }^{36}$ South Dakota School of Mines and Technology, Rapid City, South Dakota 57701, USA \\ ${ }^{37}$ Department of Physics, Southern Methodist University, Dallas, Texas 75275, USA \\ ${ }^{38}$ Department of Physics, Stanford University, Stanford, California 94305, USA \\ ${ }^{39}$ Department of Physics and Astronomy, University of Sussex, \\ Falmer, Brighton BN1 9QH, United Kingdom \\ ${ }^{40}$ Department of Physics, Syracuse University, Syracuse, New York 13210, USA \\ ${ }^{41}$ Department of Physics, University of Texas at Austin, Austin, Texas 78712, USA \\ ${ }^{42}$ Department of Physics and Astronomy, Tufts University, Medford, Massachusetts 02155, USA \\ ${ }^{43}$ Physics and Astronomy Dept., University College London, \\ Gower Street, London WC1E 6BT, United Kingdom \\ ${ }^{44}$ Department of Physics, University of Virginia, Charlottesville, Virginia 22904, USA \\ ${ }^{45}$ Department of Mathematics, Statistics, and Physics, Wichita State University, \\ Wichita, Kansas 67206, USA \\ ${ }^{46}$ Department of Physics, William \& Mary, Williamsburg, Virginia 23187, USA \\ ${ }^{47}$ Department of Physics, University of Wisconsin-Madison, Madison, Wisconsin 53706, USA
}

(Received 22 January 2020; accepted 30 April 2020; published 22 June 2020; corrected 6 May 2021)

Using the NOvA neutrino detectors, a broad search has been performed for any signal coincident with 28 gravitational wave events detected by the LIGO/Virgo Collaboration between September 2015 and July 2019. For all of these events, NOvA is sensitive to possible arrival of neutrinos and cosmic rays of $\mathrm{GeV}$ and higher energies. For five (seven) events in the NOvA Far (Near) Detector, timely public alerts from the LIGO/Virgo Collaboration allowed recording of MeV-scale events. No signal candidates were found.

DOI: $10.1103 /$ PhysRevD.101.112006

\section{INTRODUCTION}

Recent years have seen an explosion in multimessenger astronomy, and the potential for discovery continues to increase at a rapid pace. For many years, the only extrasolar source detected by more than one messenger-defined as photons, neutrinos, gravitational waves (GWs), and cosmic rays-was Supernova 1987a [1-3], seen in neutrinos as well as across the electromagnetic spectrum. With the advent of gravitational wave astronomy [4], the joint observation of GW170817 [5] with GRB 170817A [6,7]

\footnotetext{
* Corresponding author. straitm@umn.edu

Published by the American Physical Society under the terms of the Creative Commons Attribution 4.0 International license. Further distribution of this work must maintain attribution to the author(s) and the published article's title, journal citation, and DOI. Funded by SCOAP ${ }^{3}$.
}

has been added to the list. More recently, the flaring blazar TXS $0506+056$ was associated with a high-energy neutrino observed by the IceCube observatory [8].

A flux of high-energy neutrinos, of $\mathrm{GeV}$-scale and higher, is expected from any compact object merger with a neutron star remnant or merger that occurs within a significant concentration of gas [9]. Additionally, any compact object remnant would emit $\mathrm{MeV}$ neutrinos as it cooled. The merger of two neutron stars would initially produce a hot neutron star that cools primarily via neutrino emission [10]; this hot neutron star may or may not subsequently collapse to form a black hole, but produces a neutrino flux regardless. Also, gravitational waves are expected to be emitted by core-collapse supernovae, which are known neutrino sources, provided an asymmetric collapse occurs with quadrupole or higher moments [11]. Finally, gravitational waves from unknown or exotic sources (e.g., cosmic strings [12]) may be associated with neutrino bursts. Despite these possibilities, no neutrinos 
have been observed to date coincident with any gravitational wave event [13-18].

These several possibilities motivate a broad search for any detectable activity in the NOvA detectors in coincidence with gravitational wave events. Although at extragalactic distances we do not expect a detectable flux at NOvA from compact object mergers (observed by LIGO/ Virgo) or supernovae (not yet observed), it is valuable to check this hypothesis in case either a source of gravitational waves has been misidentified as extragalactic when it is not or some observable flux production mechanism has been overlooked. As NOvA has significant sensitivity to supernovalike neutrinos, we report limits on the supernovalike neutrino flux. While we search in higher-energy channels as well, NOvA's sensitivity to the usual flux models is limited compared to other observatories. As our only likely sensitivity is to the unexpected, we do not set flux limits for higher-energy signals.

\section{DETECTORS}

The NOvA experiment [19] consists of two detectors separated by $809 \mathrm{~km}$. The detector design was optimized for the detection of $\nu_{\mathrm{e}}$ appearance in a $\nu_{\mu}$ beam, specifically for the discrimination between neutral current events containing a $\pi^{0}$ and $\nu_{\mathrm{e}}$ charged current events. The requirement that the radiation length be significantly longer than a detector element set the size of the scintillator cells and motivates the use of low- $\mathrm{Z}$ materials. The NOvA detectors have been collecting data from the Fermilab Neutrinos at the Main Injector (NuMI) beam since 2013 [20,21].

The Near Detector (ND) is located underground at Fermilab, with $22 \mathrm{~m}$ water-equivalent overburden. It is designed to measure the unoscillated neutrino flux produced by Fermilab's NuMI beamline. The Far Detector (FD) is located in northern Minnesota, on the surface but slightly below grade, with a modest $3 \mathrm{~m}$ water-equivalent overburden provided by $1.3 \mathrm{~m}$ of concrete and $16 \mathrm{~cm}$ of barite. The ND is relatively small, with dimensions 16 by 4.1 by $4.1 \mathrm{~m}$ and a mass of 300 ton, while the FD has dimensions 60 by 15.6 by $15.6 \mathrm{~m}$ and a mass of $14 \mathrm{kton}$. The long axes point $28^{\circ}$ west of north; this direction is called $+z$, with the short axes $x$ and $y$ forming a right-handed coordinate system in which $+x$ is west and $+y$ is up. In the context of this search, the ND is a small, low-background detector as compared to the large, high-background FD.

The two detectors are functionally identical and consist of alternating vertical and horizontal planes of polyvinyl chloride cells [22] filled with liquid scintillator [23]. The cells are 4 by $6 \mathrm{~cm}$ and extend over the width or height of the detector. Each cell contains a single loop of wavelengthshifting fiber that extends from the readout end, down the entire length of the cell, and back to the readout end. This scheme allows for efficient light collection without the need to instrument both ends of each cell. Both ends of each fiber are coupled to a single pixel on a 32 pixel avalanche photodiode array.

The last $3 \mathrm{~m}$ of the ND is a muon range stack consisting of ten $10 \mathrm{~cm}$ thick steel plates with two scintillator planes, one horizontal and one vertical, between each steel plate. The FD has no muon range stack. With the exception of the steel plates, the detectors are $62 \%$ scintillator by mass.

Signals from each cell are continuously digitized by front-end electronics. Energy depositions over threshold are recorded for further processing. This threshold depends on position within the detectors and is typically a few $\mathrm{MeV}$. Detector-wide trigger decisions are made in a farm of Linux computers. Triggers can be issued based either on the characteristics of the data or on external signals. For instance, the energy trigger in each detector reads out candidate physics events if the total energy in a window of time exceeds a fixed value, and the NuMI trigger reads out data in a window around a time stamp received from Fermilab for each beam pulse. Data segments are available to be read out by any number of triggers independently; no trigger causes dead time for any other. The data buffer is about 30 min deep at the ND and 22 min deep at the FD, with variations caused by the number of buffer computers currently operating and the detectors' raw data rates [24].

For this analysis, the energy triggers at the ND and FD are used to collect candidate events above about 100 and $50 \mathrm{GeV}$, respectively. These triggers read out for as long as a high-energy burst continues, up to $20 \mathrm{~ms}$. At the FD, a minimum bias $10 \mathrm{~Hz}$ trigger collects either $550 \mu \mathrm{s}(97 \%$ of triggers) or $500 \mu \mathrm{s}$ (3\%; occurs when the trigger lines up with a readout block boundary) at regular $100 \mathrm{~ms}$ intervals. This trigger is used to search for events below the energy trigger's threshold, albeit with only about a $0.55 \%$ live-time fraction.

Finally, the LVC triggers at both detectors receive alerts sent by the LIGO/Virgo Collaboration (LVC) [25] over the Gamma-Ray Coordinates Network (GCN) each time a gravitational wave event candidate is detected. If such a trigger is received while the data are still available, $45 \mathrm{~s}$ of continuous data are read out, beginning $5.16 \mathrm{~s}$ before the LVC time stamp. The readout begins significantly before the LVC time stamp in order to capture a baseline for background subtraction, or conceivably correlated activity preceding the peak GW power. The precise time offset between the LVC time stamp and the beginning of NOvA readout is arbitrary and related to features of NOvA's data acquisition system (DAQ). Partial readouts can occur for triggers received when only some of the requested $45 \mathrm{~s}$ is still available; these are still analyzed (see Table I). The $45 \mathrm{~s}$ window is motivated partially by the length of time that a detectable flux of neutrinos is expected from a Galactic supernova. An even longer readout would be better for this purpose, but $45 \mathrm{~s}$ was determined to be a period that could be stably recorded by the DAQ. For signals other than nearby supernovae, we do not know of a model which 
TABLE I. NOvA's data collection for LVC events. Events beginning with GW (S) are from LVC's O1 and O2 (O3) runs. When a continuous window of data was read out in response to an LVC trigger, the number of seconds read is given. From nondetection of supernovalike neutrinos, $90 \%$ C.L. upper bounds, in units of $10^{12} \mathrm{~cm}^{-2}$, are given on the fluence for 27 and 9.6 solar mass models.

\begin{tabular}{|c|c|c|c|c|}
\hline Name & ND & FD & $\mathrm{SN}_{27 \odot}$ & $\mathrm{SN}_{9.6 \odot}$ \\
\hline GW150914 [26] & Untriggered & Bad & $\cdots$ & \\
\hline GW151012 [26] & Untriggered & No data & $\ldots$ & $\ldots$ \\
\hline GW151226 [26] & Untriggered & Untriggered & 1.9 & 5 \\
\hline GW170104 [26] & Untriggered & Untriggered & 3.3 & 10 \\
\hline GW170608 [26] & Untriggered & Untriggered & 1.5 & 2.9 \\
\hline GW170729 [26] & Untriggered & Untriggered & 2.1 & 4 \\
\hline GW170809 [26] & Untriggered & Untriggered & 2.2 & 5 \\
\hline GW170814 [26] & Untriggered & Untriggered & 4 & 10 \\
\hline GW170817 [26] & Untriggered & Untriggered & 1.4 & 3.3 \\
\hline GW170818 [26] & Untriggered & Untriggered & 1.8 & 4 \\
\hline GW170823 [26] & Untriggered & Untriggered & 2.0 & 5 \\
\hline S190408an [27] & No data & No data & $\ldots$ & $\ldots$ \\
\hline S190412m [28] & Untriggered & Untriggered & 3.3 & 9 \\
\hline S190421ar [29] & Untriggered & Untriggered & 2.7 & 6 \\
\hline S190425z [30] & Untriggered & Untriggered & 1.5 & 2.9 \\
\hline S190426c [31] & $44.1 \mathrm{~s}$ & Untriggered & 0.33 & 0.6 \\
\hline S190503bf [32] & Untriggered & Untriggered & 1.8 & 4 \\
\hline S190510g [33] & Untriggered & Untriggered & 2.4 & 4 \\
\hline S190512at [34] & Untriggered & Untriggered & 2.7 & 8 \\
\hline S190513bm [35] & $24.7 \mathrm{~s}$ & Untriggered & 0.6 & 0.9 \\
\hline S190517h [36] & Untriggered & Untriggered & 1.5 & 4 \\
\hline S190519bj [37] & Untriggered & Untriggered & 2 & 5 \\
\hline S190521g [38] & $45.0 \mathrm{~s}$ & $45.0 \mathrm{~s}$ & 0.14 & 0.32 \\
\hline S190521r [39] & Untriggered & Untriggered & 1.3 & 3.0 \\
\hline S190602aq [40] & $45.0 \mathrm{~s}$ & $45.0 \mathrm{~s}$ & 0.11 & 0.27 \\
\hline S190630ag [41] & $45.0 \mathrm{~s}$ & $45.0 \mathrm{~s}$ & 0.14 & 0.34 \\
\hline S190701ah [42] & $45.0 \mathrm{~s}$ & $45.0 \mathrm{~s}$ & 0.19 & 0.34 \\
\hline S190706ai [43] & $45.0 \mathrm{~s}$ & $17.5 \mathrm{~s}$ & 0.19 & 0.4 \\
\hline S190707q [44] & Untriggered & Untriggered & 5 & 10 \\
\hline
\end{tabular}

motivates any particular time window and which would only produce events below NOvA's energy trigger thresholds. A test $45 \mathrm{~s}$ trigger is issued each morning at 8:30 local time, both ensuring stability of the system and providing data for background estimates.

The LVC trigger has been active for the LIGO/Virgo Collaboration's "O3" run, starting in 2019 [45]; prior to that, only the energy and FD $10 \mathrm{~Hz}$ triggers were available. Each detector has recorded data from several other triggers (e.g., the NuMI trigger), but the total live time of these other triggers was negligible, and/or the characteristics of the trigger selections were unsuitable for this search.

Although not used in this analysis, we also run two triggers to collect data in the case of a Galactic supernova. One responds to an alert from Supernova Early Warning System [46], while the other is a self-trigger which responds to data collected by NOvA itself [47]. In the case in which a gravitational wave event was caused by a nearby supernova, these triggers would collect neutrino interactions in NOvA even in the absence of a LVC trigger.

\section{SEARCHES}

So as not to miss any unanticipated signals, a variety of searches were performed, each designed to be as generic as possible. The energy and $10 \mathrm{~Hz}$ triggers were used to search for any burst within a $1000 \mathrm{~s}$ window centered on an LVC event time stamp in addition to the $45 \mathrm{~s}$ readout from the LVC triggers. We searched for bursts of 1) events selected by energy from several $\mathrm{MeV}$ to many $\mathrm{TeV}$ without regard for detailed event characteristics, 2) contained GeV-scale events, and 3) events with tracks, further broken down into several categories.

\section{A. Energy searches}

\section{Supernovalike}

First, a search was performed for events similar to those expected to be caused by $\mathrm{O}(10 \mathrm{MeV})$ supernova neutrinos. NOvA is primarily sensitive to $\bar{\nu}_{\mathrm{e}}$ through the inverse beta decay channel, with $75 \%$ of interactions expected through this channel in the no-oscillation case. We have some sensitivity to $\nu_{\mathrm{e}}$ via $\nu_{\mathrm{e}}{ }^{12} \mathrm{C} \rightarrow \mathrm{e}^{-12} \mathrm{~N}$ (5\% of interactions) and any flavor through electron elastic scattering and excitation of carbon nuclei (20\%).

This selection was optimized separately for the ND and FD and was designed to maximize the signal significance $S / \sqrt{B}$, where the simulated signal, $S$, used for the optimization procedure was generated using the Garching supernova flux [48] with the GENIE Monte Carlo generator [49]. The background, $B$, was determined from minimum bias data.

To select activity as supernovalike, first, all tracks (which are mainly cosmic ray muons) and other $\mathrm{GeV}$-scale clusters of activity are removed, as is any hit within 14 planes or 28 cells of such activity and within a time window extending from $2 \mu$ s before to $13 \mu$ s after the $\mathrm{GeV}$-scale activity. This effectively removes any hits that were associated with the GeV-scale activity but not identified as such by the clustering algorithm, as well as removes all Michel electrons from muon decay. For highly energetic cosmic ray events, the time cut is extended from 13 to $200 \mu$ s, which removes neutron capture activity and spurious hits caused by electronics effects. Even with the high background level in the FD, which is on the surface, these simple cuts remove only $11 \%$ of the signal.

Further, hits near the top or sides of each detector are removed. Hits must be at least 50 (20) cells from the top of the detector in the FD (ND), 10 (4) cells from the east and west sides of the detector, and 2 (4) planes from the north and south ends. Hits of very low and very high energy are removed to eliminate noise and activity in excess of 
supernovalike energy. Once individual hits are selected in this manner, candidate events are formed from hit pairs consisting of one hit in a horizontal plane and one in an adjacent vertical plane. Given the $z$ positions of the planes, the $x$ position of the vertical cell, and the $y$ position of the horizontal cell, the three-dimensional position of the cluster is determined. These hits must have times within $250 \mathrm{~ns}$ of each other after correcting the timing of each hit using the coordinate provided by the other and the propagation speed of light in the detector.

For both detectors, this selection has $20 \%$ efficiency for supernovalike inverse beta decay events within the accepted volume. The background rate is $450 \mathrm{~Hz}(0.5 \mathrm{~Hz})$ at the FD (ND). While the FD background is large, the expected peak rate of selected events for a supernova in the Galactic core is $\sim 4 \mathrm{kHz}$, which makes such a signal easily observable. Extragalactic sources associated with gravitational wave events, however, would need to be substantially brighter in supernovalike neutrinos to be seen by NOvA.

\section{Subsupernovalike}

To search for lower-energy signals in the range of 1-10 MeV, we run two similar selections. Each is the same as the supernovalike selection above, except that any hit selected as part of a supernovalike event is removed from consideration (to create a statistically independent sample), and individual hits are selected instead of pairs. In the first of these selections, the low-energy requirement for hits is lowered to just above the level associated with avalanche photodiode noise, equivalent to a few MeV. In the other, there is no low-energy requirement.

Without the requirement that events include a hit in each view, three-dimensional locations of candidates cannot be reconstructed, making it impossible to know when a hit is near the end of a cell and therefore near an edge of the detector, increasing the cosmogenic background. Natural radioactivity is also selected-the NOvA design made no attempt at radiopurity. Both of these considerations increase the background rates dramatically. The background rate at the FD (ND) is $42 \mathrm{MHz}(190 \mathrm{kHz})$ for the selection without a low-energy cut and $550 \mathrm{kHz}$ $(38 \mathrm{kHz})$ with most electronics noise excluded by the low-energy cut.

In the case of gravitational wave events for which we received a LVC trigger and read out a period of continuous data, usually $45 \mathrm{~s}$, these three $\mathrm{MeV}$-scale searches (supernovalike, subsupernovalike with a low-energy cut, and subsupernovalike without a low-energy cut) were run separately both for the continuous LVC-triggered data and for $1000 \mathrm{~s}$ of $10 \mathrm{~Hz}$ trigger data in the FD. While these data streams are not entirely disjoint, only $0.55 \%$ of the LVC-triggered data are also present in the $10 \mathrm{~Hz}$ trigger data, so we neglect the overlap.

\section{High-energy Far Detector events}

At the FD, the energy trigger is used to search for any excess of events depositing $50 \mathrm{GeV}$ or higher. Besides examining the trigger rate, six selections are made to select higher-energy events with two general topologies. The energy trigger selects events in which the majority of the energy is deposited promptly and also integrates the deposited energy up to $20 \mathrm{~ms}$ to select periods of time with a large total activity. Three selections are made of events in which the energy appears within a single $50 \mu$ s time window, with the requirements of an energy deposition of at least $200 \mathrm{GeV}, 2 \mathrm{TeV}$, and $20 \mathrm{TeV}$ for the first, second, and third selections, respectively. A second set of three selections allows the energy to arrive over a longer period of time - up to $20 \mathrm{~ms}$ - with total energy depositions of at least $400 \mathrm{GeV}, 4 \mathrm{TeV}$, and $40 \mathrm{TeV}$.

\section{B. Contained events}

FD data were examined for any contained activity. Such activity would be indicative of neutrino interactions in the $\mathrm{GeV}$ to tens-of-GeV range; however, in this search, no neutrinolike requirements were imposed on the event topology. To be considered a contained event, all hits of a cluster must be at least $130 \mathrm{~cm}$ from the bottom, east, and west faces of the detector; at least $75 \mathrm{~cm}$ from the north and south faces; and at least $280 \mathrm{~cm}$ from the top. The cluster must have at least ten hits. The number of planes between the northernmost and southernmost hits in horizontal planes must be at least 9 , with the same requirement made of the vertical planes. These requirements eliminate most cosmic ray activity. Furthermore, for both views, the occupancy within the smallest rectangular box that surrounds all the hits must be at least $2 \%$, to prevent the selection of uncorrelated low-energy activity, and no more than $10 \%$, to eliminate classes of electronics noise which cause spurious hits in many adjacent channels.

The efficiency of this selection depends on assumptions about the origin of a potential signal. Most notably, physically larger events will be selected with lower efficiency because they are more often near the edges. Some loss of efficiency also occurs because background cosmic ray activity can overlap in time and space with signal events, causing them to appear uncontained. For few-GeV neutrinolike events, this effect reduces the efficiency by only a few percent. Similar considerations apply to the fully and partially contained track selections below.

\section{Track selections}

In each detector, the time distribution of tracks is examined with tracks selected in nine ways, using all combinations of three track topologies and three pointing requirements. The three topologies are fully contained tracks, tracks which start or stop in the detector, and an inclusive selection of any kind of track. In each case, counts 
are made 1) without regard to pointing, 2) with the requirement of pointing to the LVC $90 \%$ allowed region convolved with a $1.3^{\circ}$ resolution, and 3 ) the same but with a $16^{\circ}$ resolution. Convolution was performed with HEALPIX [50], which provides functions for analysis of binned data on a sphere. The background rate of events in the most inclusive category - any type of track with any pointing is $110 \mathrm{kHz}(36 \mathrm{~Hz})$ at the FD (ND). When multiple tracks are detected in coincidence, they are counted as a single event with the least-contained track setting the category. This procedure ensures that the background rate closely follows a Poisson distribution.

The $1.3^{\circ}$ convolution represents an estimate of NOvA's track pointing resolution, and so this selection generically represents hypotheses that would cause charged particles that appear in NOvA to point directly back at the gravitational wave source, or nearly so. The most likely scenario would be detection of a part of a high-energy neutrino interaction in the atmosphere or rock surrounding the detector. The $16^{\circ}$ convolution is meant to select secondary charged particle tracks resulting from lower-energy interactions and was set to represent the approximate range of reconstructed muon angles resulting from $10 \mathrm{GeV} \nu_{\mu}$ interactions in or near the detector. Despite these motivations, the selections are meant to be as generic as reasonably possible and do not assume any particular interaction model.

In addition, in the FD only, upward-going muon tracks are selected. Because of how light propagates in the long cells in NOvA's design, nanosecond-level timing is not available for individual hits, and therefore track direction is difficult to determine for short tracks. At the FD, track direction can be determined for tracks over $8 \mathrm{~m}$ by fitting the timing distribution under the upward and downward hypotheses. This method is used to select upward-going muons, a potential signal of $\nu_{\mu}$ interactions either in the detector or the rock beneath it. As with the other track selections, this is repeated using the allowed sky regions convolved with $1.3^{\circ}$ and $16^{\circ}$ resolutions.

\section{NUMI BEAM VETO}

The ND is exposed to Fermilab's NuMI neutrino beam, which provides $10 \mu \mathrm{s}$ long pulses of $\nu_{\mu}$ or $\bar{\nu}_{\mu}$ with a mean energy of $2.7 \mathrm{GeV}$ every 1.3 to $1.4 \mathrm{~s}$ during beam operations. Several neutrino interactions are typically recorded in each beam pulse. All hits recorded from the beginning of each pulse to $3 \mathrm{~ms}$ afterward are eliminated from this analysis. This time interval is sufficient to allow all significant effects of the neutrino interactions to end. The longest-lived such effect is caused by neutrons produced in the surrounding rock, which can thermalize in the rock, then travel through the air in the detector hall for several meters at $\sim 225 \mathrm{~m} / \mathrm{s}$ before arriving at the detector and being captured with a characteristic time of $50 \mu \mathrm{s}$. While beam interactions also produce radioactive isotopes, including ${ }^{12} \mathrm{~B}$ and ${ }^{12} \mathrm{~N}$, the rate of their decays is small compared to the background rate, so they do not motivate a longer beam exclusion window.

The NuMI interaction rate at the FD of $\mathrm{O}(1)$ interaction per day is negligible. Likewise, the ND is also exposed to Fermilab's Booster Neutrino Beam but is far enough off axis to yield an event rate of about 1.5 per day, and this rate is also neglected.

\section{DATASET}

Table I shows a summary of NOvA data collected for each of the gravitational wave events reported by the LIGO/ Virgo Collaboration from GW150914, the first detection, in September 2015, through S190707q, the last event analyzed in this report. As a result of receiving prompt triggers from LVC, low-energy data were recorded with good efficiency for five (seven) events in the FD (ND). Alerts must be received within 10 min to ensure a full readout. For S190426c and S190513bm, the trigger was received sufficiently late (25 and $28 \mathrm{~min}$, respectively) that only

TABLE II. Summary of each trigger stream, selection, and background method. The five methods of determining background are described in the text.

\begin{tabular}{ll}
\hline \hline Trigger, selection & \multicolumn{1}{c}{ Background method } \\
\hline FD LVC, 10 Hz: & \\
Supernovalike & Flat, self-determined \\
Subsupernova & Flat, self-determined non-Poisson \\
Total tracks & Flat, self-determined \\
$\ldots$ any pointing & Varying, self-determined \\
Stopping tracks & Flat, self-determined \\
$\ldots 16^{\circ}$ pointing & Varying, self-determined \\
$\ldots 1.3^{\circ}$ pointing & Varying, uncorrelated samples \\
Contained tracks & Flat, uncorrelated samples \\
$\ldots$ any pointing & Varying, uncorrelated samples \\
Upward tracks & Flat, uncorrelated samples \\
$\ldots$ any pointing & Varying, uncorrelated samples \\
Contained activity & Flat, uncorrelated samples \\
FD energy trigger: & \\
50,400 GeV & Flat, self-determined \\
4,40 TeV & Flat, uncorrelated samples \\
200 GeV prompt & Flat, self-determined \\
2,20 TeV prompt & Flat, uncorrelated samples \\
ND LVC trigger: & \\
Supernovalike & Flat, uncorrelated samples \\
Subsupernova & Flat, self-determined \\
ND energy trigger: & \\
Total tracks & Varying, self-determined \\
$\ldots 16^{\circ}$ pointing & Varying, self-determined \\
$\ldots 1.3^{\circ}$ pointing & Varying, uncorrelated samples \\
Stopping tracks & Flat, uncorrelated samples \\
$\ldots$ any pointing & Varying, uncorrelated samples \\
Contained tracks & Flat, uncorrelated samples \\
$\ldots$ any pointing & Varying, uncorrelated samples \\
Contained activity & Flat, uncorrelated samples \\
\hline \hline & \\
\hline &
\end{tabular}


partial readout was possible at the ND. For S190706ai, full ND readout was possible given the 18 min trigger latency, but only a partial FD readout was possible. A prompt alert was sent for S190521r, but our connection to GCN was down at the time. In the remaining cases, data including only low-energy events were no longer available when the trigger arrived.

High-energy data, along with low-energy data with $0.55 \%$ live time, were taken with the full $1000 \mathrm{~s}$ window around the gravitational wave time stamp for all other events with the exceptions of 1) S190408an, for which both detectors were down; 2) GW151012, for which the FD was down; and 3) GW150914, for which the FD was taking data but suffering DAQ instability (we do not use such data here).

\section{ANALYSIS}

For each selection described above, we searched for any excess in $1 \mathrm{~s}$ bins during the $1000 \mathrm{~s}$ analysis window, or the $45 \mathrm{~s}$ trigger window for LVC-triggered data streams. The bin width is intended to be similar to the duration of the initial pulse of neutrinos from a supernova as well as to that of a short gamma ray burst, such as that detected along with GW170817 [6,7], while not being finely tuned to any particular model. Different strategies are used to determine background level, depending on the characteristics of each sample. These are described below and summarized in Table II.

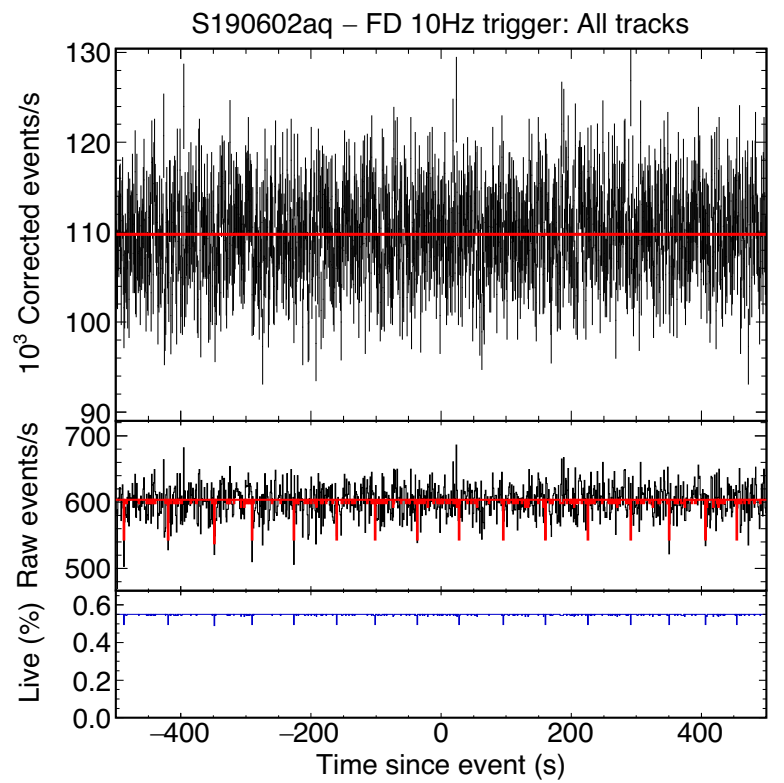

FIG. 1. Rate of tracks observed in the FD $10 \mathrm{~Hz}$ trigger in the $1000 \mathrm{~s}$ around S190602aq. The top panel shows the inferred total rate of tracks in each second (raw rate divided by integrated live time), the middle panel shows the number of tracks actually observed, and the bottom panel shows the integrated live time. A fit to a constant rate is shown. Error bars in all plots are statistical.
For several selections, the background level is many hertz. In these cases, we measured the background directly as the mean rate in the analysis window, assuming that no burst of astrophysical activity will be both large enough and long enough-well over $\mathrm{O}(100 \mathrm{~s})$ and spanning the time from before to after the gravitational wave burst-to significantly skew that mean. The rate of all tracks is an example of this class of selections, as shown in Fig. 1.

For all high-background selections with the exception of the few-MeV subsupernovalike searches, the background
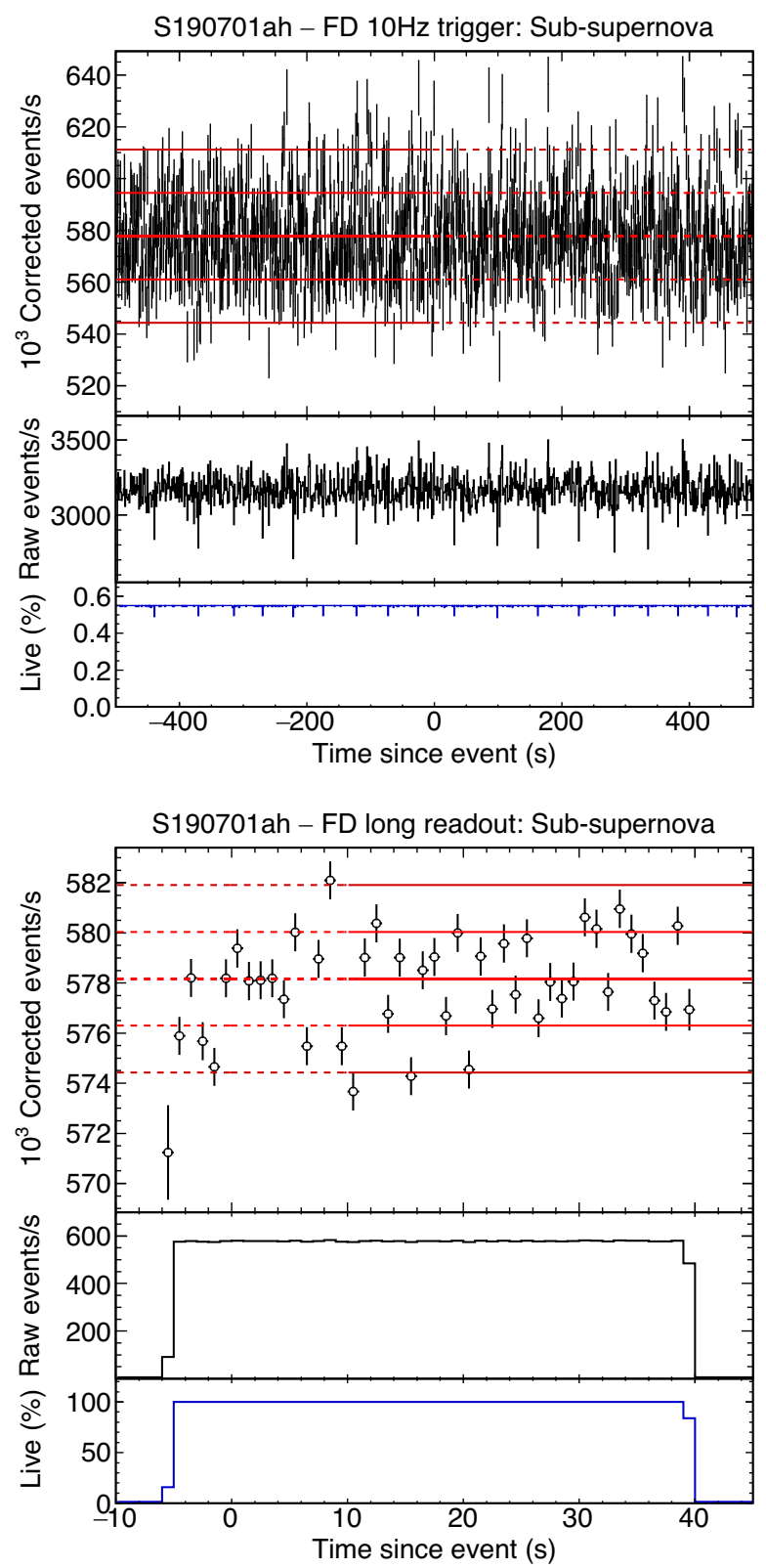

FIG. 2. Results of the subsupernova event search in the FD for S190701ah, using a low-energy cut. Top: the $10 \mathrm{~Hz}$ trigger. The three panes have the same meanings as in Fig. 1. Bottom: the LVC trigger with $100 \%$ live time for $45 \mathrm{~s}$. The lines show the measured background rate (center line) with 1 and $2 \sigma$ extents, with the control region solid and signal region dashed. 
events are nearly all uncorrelated, such that an excess can be quantified using Poisson statistics. For the few-MeV samples, many events are correlated-for instance, bursts of neutron captures from air showers-so instead we measure the Gaussian width of the distribution of bin contents in a control region to determine the significance of excesses in a signal region. For the $1000 \mathrm{~s}$ windows, the control region is defined as beginning $500 \mathrm{~s}$ before the gravitational wave event time stamp and ending $5 \mathrm{~s}$ before. From there to $500 \mathrm{~s}$ after is the signal region. For the $45 \mathrm{~s}$ readouts, the control sample is defined as 10 to $40 \mathrm{~s}$ after the event, with the assumption that interesting activity is more likely in the first $10 \mathrm{~s}$. Examples of each of these are shown in Fig. 2.

In the case of high-background samples with a restricted sky region, the background changes over time because the allowed region's zenith angle is changing and the cosmic ray flux is a function of zenith angle. Although the precise form, as a function of time, of the background rate is quite complex, for the relatively short time windows used in this analysis, we found it sufficient to fit a linear function to the observed rate (see Fig. 3).

For low-background samples without pointing dependence, the background level was determined by counting selected events in many uncorrelated time windows of the same trigger stream. For instance, the rate of FD events over $2 \mathrm{TeV}$ was determined to be $1.0 \times 10^{-3} \mathrm{~Hz}$. Likewise, this strategy is used for the supernovalike event search in the ND (see Fig. 4).

Finally, for low-background samples restricted by the LVC $90 \%$ allowed region, we use an ensemble of data with

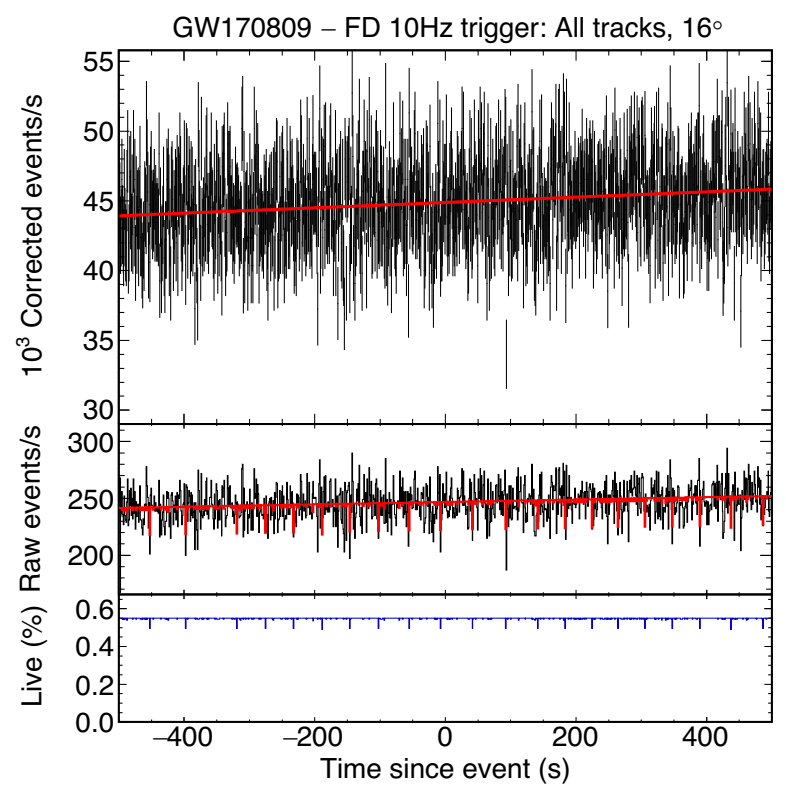

FIG. 3. Rate of tracks observed around GW170809 within the $90 \%$ C.L. sky region after $16^{\circ}$ resolution is applied, illustrating the linear background fit used for time-varying selections. The three panes have the same meanings as in Fig. 1.

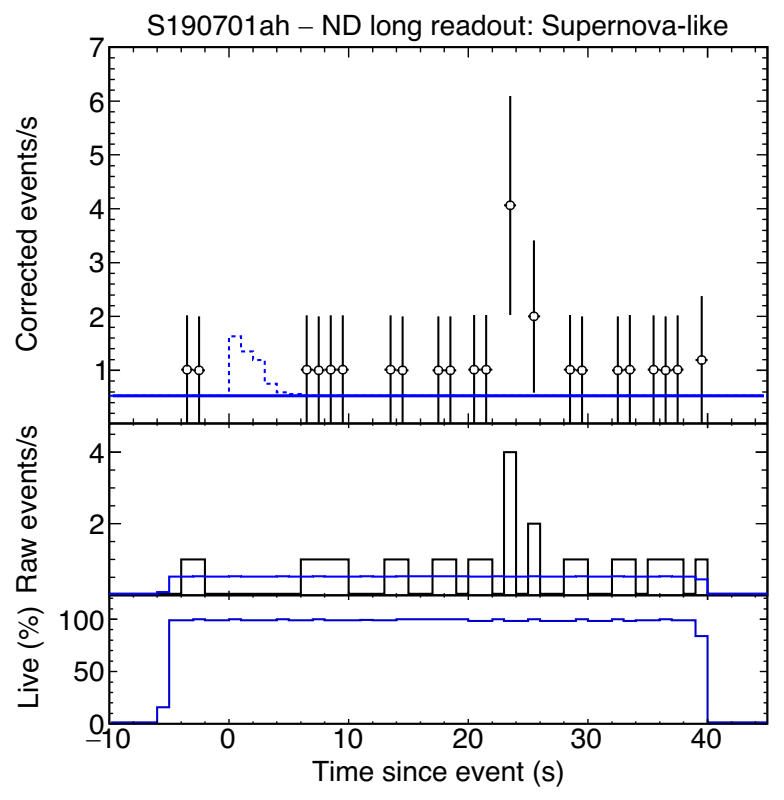

FIG. 4. Supernovalike event search for S190701ah, in the ND, using the LVC trigger. The background rate, shown as a solid line, is determined from a large number of uncorrelated time windows. The dashed line shows the expected signal for a 9.6 solar mass supernova at $10 \mathrm{kpc}$. The bin with four events has $p=9 \%$ taking into account the trials factor of the 45 bins in this plot alone, and is not considered significant.

uncorrelated time stamps and select events using the same sky region, in zenith and azimuth, as the signal event (see, e.g., Fig. 5).

The significance of excesses was quantified by taking into account the trials factor given the number of bins searched for each gravitational wave event. Potentially interesting excesses in the first $10 \mathrm{~s}$ after a gravitational wave event were considered special and only subject to a trials factor counting other bins within the first $10 \mathrm{~s}$. Each gravitational wave event was considered separately with no statistics computed using the ensemble of events.

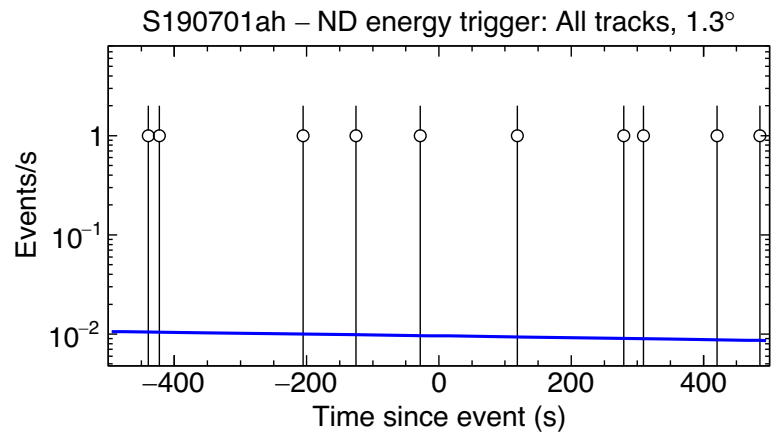

FIG. 5. A search in the ND for a burst of tracks, regardless of containment, around S190701ah, that point to the LVC 90\% C.L. region convolved with $1.3^{\circ}$ resolution. The time-varying background rate (dashed line) is shown. In this $1000 \mathrm{~s}$ window, there are ten events on a background of 9.6. 

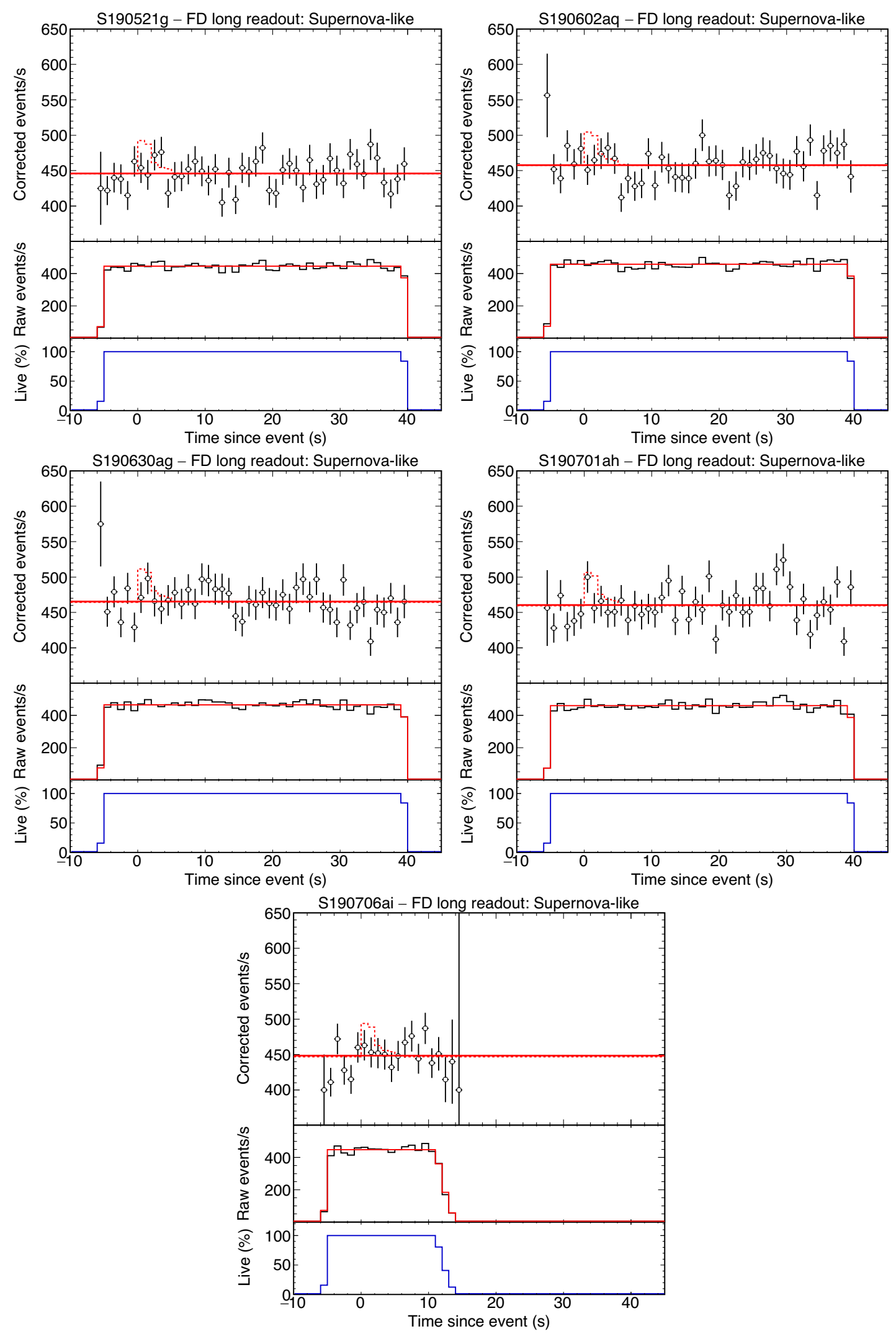

FIG. 6. Results of supernovalike neutrino search for events with FD LVC-triggered data. The readout of S190706ai is incomplete due to a late trigger, with progressively less data available each second between 11 and 14 s. A fit to a constant rate is shown as a solid red line in the upper and middle panes of each plot. Dashed lines show best fits for a 9.6 solar mass supernova at $10 \mathrm{kpc}$. 
We used a blind analysis, defining "significant" as being at least $3 \sigma$ over background after the trials factor. No significant excesses were found. Post hoc visual inspection of the time distributions also did not reveal any features other than those expected in background.

Given NOvA's sensitivity to several-MeV neutrinos, but relatively small acceptance for higher-energy events, the selections most likely to have shown a positive signal are the LVC-triggered supernovalike searches. Figure 6 shows the five gravitational wave events for which an LVCtriggered readout window is available in the FD. None reveals any evidence of a supernovalike burst.

\section{A. Supernovalike neutrino fluence limits}

Since no significant excesses were found in searches for a supernovalike signal, we set limits on the neutrino fluence. We assume the Garching models for 27 and 9.6 solar mass stars, without neutrino oscillations. Neutrino oscillations and other flavor-changing effects will modify the signal in NOvA, and can either increase or decrease the observed interaction rate [48]. We assume that a potential supernova neutrino burst would occur in coincidence with the GW burst.

The 27 solar mass model predicts a larger flux with a higher mean neutrino energy and a time distribution more strongly peaked in the first second. The higher-energy neutrinos are more efficiently detected, particularly by the Far Detector, leading to stronger limits as compared to the 9.6 solar mass model. The differing time distributions between the models, combined with background fluctuations, means the limits obtained for the two models are not simply proportional.

We perform a Bayesian analysis with a flat prior in neutrino fluence, profiling over the background level in the case of the FD (in the ND, the background is fixed using uncorrelated time windows; see Sec. VI). Limits are shown in Table I. For the case in which we read out both detectors in response to an LVC trigger, the mean $90 \%$ C.L. upper limit on neutrino fluence is $0.12(0.24) \times 10^{12} \mathrm{~cm}^{-2}$ for the 27 (9.6) solar mass model. When only FD $10 \mathrm{~Hz}$ trigger data are available, the mean limits are $1.5(4) \times 10^{12} \mathrm{~cm}^{-2}$.

An upper limit on fluence, $F_{90}$, can be converted to a lower limit on the distance, $r_{90}$, to a hypothetical supernova,

$$
r_{90}=\sqrt{\frac{N}{4 \pi F_{90}}},
$$

where $N$ is the total number of neutrinos emitted. For the Garching 27 (9.6) solar mass model, $N=11(6.8) \times 10^{57}$.
For all events with LVC-triggered readout in both detectors, distances closer than $22 \mathrm{kpc}$ are excluded at $90 \%$ C.L. for the case of a 27 solar mass supernova. This limit varies by event and is as far as $29 \mathrm{kpc}$ for S190602aq. These limits exclude $99 \%$ of the volume in which potential supernovae could occur in the Milky Way [51]. For the 9.6 solar mass model, distances up to $12 \mathrm{kpc}$ are excluded in all cases for which we have LVC-triggered readout, with up to $15 \mathrm{kpc}$ excluded for S190602aq. These exclusions cover $60 \%-80 \%$ of potential Galactic supernovae. Even for events in which only FD $10 \mathrm{~Hz}$ trigger data are available, 2.4-4 (4-8) kpc are excluded in the 9.6 (27) solar mass case, covering $4 \%-12 \%(12 \%-34 \%)$ of the galaxy.

\section{CONCLUSION}

The NOvA detectors, which have sensitivity to signals, particularly neutrinos, in the $\mathrm{MeV}-\mathrm{TeV}$ range, detected no significant excesses of events during the time around any of 28 gravitational wave events reported by the LIGO/Virgo Collaboration from September 2015 through July 2019 for which at least one NOvA detector was active. Sensitivity to MeV-scale events was best for S190521g, S190602aq, S190630ag, and S190701ah, all binary black hole mergers during which we recorded $45 \mathrm{~s}$ of continuous data in both detectors.

The NOvA Collaboration intends to continue operating both detectors and receiving LVC triggers through 2025 .

\section{ACKNOWLEDGMENTS}

This document was prepared by the NOvA Collaboration using the resources of the Fermi National Accelerator Laboratory (Fermilab), a U.S. Department of Energy, Office of Science, High Energy Physics(HEP) User Facility. Fermilab is managed by Fermi Research Alliance, LLC, acting under Contract No. DE-AC0207CH11359. This work was supported by the U.S. Department of Energy; the U.S. National Science Foundation; the Department of Science and Technology, India; the European Research Council; the MSMT CR, GA UK, Czech Republic; the RAS, RFBR, Ministry of Education and Science of the Russian Federation (RMES), RSF, and BASIS Foundation, Russia; CNPq and FAPEG, Brazil; STFC and the Royal Society, United Kingdom; and the state and University of Minnesota. We are grateful for the contributions of the staffs of the University of Minnesota at the Ash River Laboratory and of Fermilab. 
[1] K. Hirata et al. (Kamiokande-II Collaboration), Observation of a Neutrino burst from the Supernova SN 1987a, Phys. Rev. Lett. 58, 1490 (1987).

[2] R. M. Bionta, G. Blewitt, C. B. Bratton, D. Casper, A. Ciocio, R. Claus et al., Observation of a Neutrino Burst in Coincidence with Supernova SN 1987a in the Large Magellanic Cloud, Phys. Rev. Lett. 58, 1494 (1987).

[3] E. N. Alekseev, L. N. Alekseeva, V. I. Volchenko, and I. V. Krivosheina, Possible detection of a neutrino signal on 23 February 1987 at the Baksan underground scintillation telescope of the institute of nuclear research, JETP Lett. 45, 589 (1987), https://inspirehep.net/record/255616.

[4] B. P. Abbott et al. (Virgo, LIGO Scientific Collaborations), Observation of Gravitational Waves from a Binary Black Hole Merger, Phys. Rev. Lett. 116, 061102 (2016).

[5] B. P. Abbott et al. (Virgo, LIGO Scientific Collaborations), GW170817: Observation of Gravitational Waves from a binary Neutron Star Inspiral, Phys. Rev. Lett. 119, 161101 (2017).

[6] A. Goldstein et al., An ordinary short gamma-ray burst with extraordinary implications: Fermi-GBM detection of GRB 170817A, Astrophys. J. 848, L14 (2017).

[7] B. P. Abbott et al. (LIGO Scientific, Virgo, Fermi-GBM, INTEGRAL Collaborations), Gravitational waves and gamma-rays from a binary neutron star merger: GW170817 and GRB 170817A, Astrophys. J. 848, L13 (2017).

[8] M. G. Aartsen et al. (IceCube, Fermi-LAT, MAGIC, AGILE, ASAS-SN, HAWC, H.E.S.S., INTEGRAL, Kanata, Kiso, Kapteyn, Liverpool Telescope, Subaru, Swift NuSTAR, VERITAS, VLA/17B-403 Collaborations), Multimessenger observations of a flaring blazar coincident with high-energy neutrino IceCube-170922A, Science 361, eaat1378 (2018).

[9] N. Fraija, MeV-GeV neutrino propagation as a signal of magnetic field amplification in neutron star merger, J. High Energy Astrophys. 11-12, 29 (2016).

[10] F. Foucart, R. Haas, M. D. Duez, E. O’Connor, C. D. Ott, L. Roberts, L. E. Kidder, J. Lippuner, H. P. Pfeiffer, and M. A. Scheel, Low mass binary neutron star mergers: gravitational waves and neutrino emission, Phys. Rev. D 93, 044019 (2016).

[11] B. P. Abbott et al. (LIGO Scientific, Virgo Collaborations), A first targeted search for gravitational-wave bursts from core-collapse supernovae in data of first-generation laser interferometer detectors, Phys. Rev. D 94, 102001 (2016).

[12] Y. Cui, M. Lewicki, D. E. Morrissey, and J. D. Wells, Cosmic archaeology with gravitational waves from cosmic strings, Phys. Rev. D 97, 123505 (2018).

[13] M. Agostini et al., A search for low-energy neutrinos correlated with gravitational wave events GW150914, GW151226 and GW170104 with the Borexino detector, Astrophys. J. 850, 21 (2017).

[14] A. Gando et al. (KamLAND Collaboration), Search for electron antineutrinos associated with gravitational wave events GW150914 and GW151226 using KamLAND, Astrophys. J. 829, L34 (2016).

[15] K. Abe et al. (Super-Kamiokande Collaboration), Search for neutrinos in Super-Kamiokande associated with gravitational wave events GW150914 and GW151226, Astrophys. J. 830, L11 (2016).

[16] S. Adrian-Martinez et al. (Virgo, IceCube, ANTARES, LIGO Scientific Collaborations), High-energy neutrino follow-up search of gravitational wave event GW150914 with Antares and IceCube, Phys. Rev. D 93, 122010 (2016).

[17] A. Albert et al. (Virgo, IceCube, ANTARES, LIGO Scientific Collaborations), Search for high-energy neutrinos from gravitational wave event GW151226 and candidate LVT151012 with ANTARES and IceCube, Phys. Rev. D 96, 022005 (2017).

[18] A. Aab et al. (Pierre Auger Collaboration), Ultrahighenergy neutrino follow-up of gravitational wave events GW150914 and GW151226 with the Pierre Auger Observatory, Phys. Rev. D 94, 122007 (2016).

[19] D. S. Ayres et al. (NOvA Collaboration), The NOvA Technical Design Report, FERMI-LAB-DESIGN-200701, 2007.

[20] P. Adamson et al., The NuMI neutrino beam, Nucl. Instrum. Methods A 806, 279 (2016).

[21] M. A. Acero et al. (NOvA Collaboration), First Measurement of Neutrino Oscillation Parameters Using Neutrinos and Antineutrinos by NOvA, Phys. Rev. Lett. 123, 151803 (2019).

[22] R. L. Talaga et al., PVC extrusion development and production for the NOvA neutrino experiment, Nucl. Instrum. Methods A 861, 77 (2017).

[23] S. Mufson et al., Liquid scintillator production for the NOvA experiment, Nucl. Instrum. Methods A 799, 1 (2015).

[24] A. Norman et al., Performance of the NOvA data acquisition and trigger systems for the full $14 \mathrm{kT}$ far detector, J. Phys. Conf. Ser. 664, 082041 (2015).

[25] B. P. Abbott et al., Prospects for observing and localizing gravitational-wave transients with Advanced LIGO, Advanced Virgo and KAGRA, Living Rev. Relativity 21, 3 (2018).

[26] B. P. Abbott et al. (LIGO Scientific, Virgo Collaborations), GWTC-1: A Gravitational-Wave Transient Catalog of Compact Binary Mergers Observed by LIGO and Virgo During the First and Second Observing Runs, Phys. Rev. X 9, 031040 (2019).

[27] LIGO and Virgo Scientific Collaborations, LIGO, Virgo S190408an: Identification of a GW binary merger candidate, GCN Circular No. 24069 (2019).

[28] LIGO and Virgo Scientific Collaborations, LIGO, Virgo S190412m: Identification of a GW binary merger candidate, GCN Circular No. 24098 (2019).

[29] LIGO and Virgo Scientific Collaborations, LIGO, Virgo S190421ar: Identification of a GW binary merger candidate, GCN Circular No. 24141 (2019).

[30] LIGO and Virgo Scientific Collaborations, LIGO, Virgo S190425z: Identification of a GW binary merger candidate, GCN Circular No. 24168 (2019).

[31] LIGO and Virgo Scientific Collaborations, LIGO, Virgo S190426c: Identification of a GW binary merger candidate, GCN Circular No. 24237 (2019).

[32] LIGO and Virgo Scientific Collaborations, LIGO, Virgo S190503bf: Identification of a GW binary merger candidate, GCN Circular No. 24377 (2019). 
[33] LIGO and Virgo Scientific Collaborations, LIGO, Virgo S190510g: Identification of a GW binary merger candidate, GCN Circular No. 24442 (2019).

[34] LIGO and Virgo Scientific Collaborations, LIGO, Virgo S190512at: Identification of a GW binary merger candidate, GCN Circular No. 24503 (2019).

[35] LIGO and Virgo Scientific Collaborations, LIGO, Virgo S190513bm: Identification of a GW binary merger candidate, GCN Circular No. 24522 (2019).

[36] LIGO and Virgo Scientific Collaborations, LIGO, Virgo S190517h: Identification of a GW binary merger candidate, GCN Circular No. 24570 (2019).

[37] LIGO and Virgo Scientific Collaborations, LIGO, Virgo S190519bj: Identification of a GW binary merger candidate, GCN Circular No. 24598 (2019).

[38] LIGO and Virgo Scientific Collaborations, LIGO, Virgo S190521g: Identification of a GW binary merger candidate, GCN Circular No. 24621 (2019).

[39] LIGO and Virgo Scientific Collaborations, LIGO, Virgo S190521r: Identification of a GW binary merger candidate, GCN Circular No. 24632 (2019).

[40] LIGO and Virgo Scientific Collaborations, LIGO, Virgo S190602aq: Identification of a GW binary merger candidate, GCN Circular No. 24717 (2019).

[41] LIGO and Virgo Scientific Collaborations, LIGO, Virgo S190630ag: Identification of a GW binary merger candidate, GCN Circular No. 24922 (2019).

[42] LIGO and Virgo Scientific Collaborations, LIGO, Virgo S190701ah: Identification of a GW binary merger candidate, GCN Circular No. 24950 (2019).

[43] LIGO and Virgo Scientific Collaborations, LIGO, Virgo S190706ai: Identification of a GW binary merger candidate, GCN Circular No. 24998 (2019).
[44] LIGO and Virgo Scientific Collaborations, LIGO, Virgo S190707q: Identification of a GW binary merger candidate, GCN Circular No. 25012 (2019).

[45] B. P. Abbott et al. (KAGRA, LIGO Scientific, VIRGO Collaborations), Prospects for observing and localizing gravitational-wave transients with Advanced LIGO, Advanced Virgo and KAGRA, Living Rev. Relativity 21, 3 (2018).

[46] P. Antonioli et al., The supernova early warning system, New J. Phys. 6, 114 (2004).

[47] J. A. Vasel, A. Sheshukov, and A. Habig (NOvA Collaboration), Observing the next galactic supernova with the NOvA detectors, in Proceedings, Meeting of the APS Division of Particles and Fields (DPF 2017) at Fermilab (SLAC Electronic Conference Proceedings Archive, Menlo Park, CA, 2017).

[48] A. Mirizzi et al., Supernova neutrinos: Production, oscillations and detection, Riv. Nuovo Cimento 39, 1 (2016).

[49] C. Andreopoulos et al., The GENIE neutrino Monte Carlo generator, Nucl. Instrum. Methods A 614, 87 (2010).

[50] K. M. Górski, E. Hivon, A. J. Banday, B. D. Wandelt, F. K. Hansen, M. Reinecke, and M. Bartelmann, HEALPix: A framework for high-resolution discretization and fast analysis of data distributed on the sphere, Astrophys. J. 622, 759 (2005).

[51] A. Mirizzi, G. G. Raffelt, and P. D. Serpico, Earth matter effects in supernova neutrinos: optimal detector locations, J. Cosmol. Astropart. Phys. 05 (2006) 012.

Correction: Columns 4 and 5 in Table I were incorrect and have been fixed. The distance values and the last percentage range in the last paragraph of Sec. VI were also incorrect and have been fixed. 